\section{BDNF: an indicator of}

\section{insomnia?}

Molecular Psychiatry (2014) 19, 151-152; doi:10.1038/mp.2013.10;

published online 12 February 2013

In the last decade, brain-derived neurotrophic factor (BDNF) has become increasingly accepted as a central mediator of the effects of stress on neuronal plasticity and its implication for psychopathology. Neurotrophic functions of BDNF are implicated with neuronal survival, learning, memory, appetite and sleep. ${ }^{1,2}$ The neurotrophin hypothesis is based on these features and proposes that stress-related mental disorders result from stress-induced decreases in BDNF expression. ${ }^{3}$ Sleep problems are common features in many stress-related mental disorders, problems that may lead to impairment of physical and mental health because sleep loss is often followed by higher stress vulnerability. ${ }^{4}$ Thus, insomnia is very common among depressed patients. ${ }^{5}$ Of note, in accordance with the 'neurotrophin hypothesis of depression', BDNF seems to be involved in major depression and antidepressant action. ${ }^{6,7}$ Although a majority of studies have concentrated on specifying the role of BDNF in depression, the relation between BDNF and insomnia has not been a focus of recent research.

Here we investigated serum BDNF levels of adults with current symptoms of insomnia and non-sleep disturbed controls. The study was approved by the local ethics committee of the University of Basel. The sample pool consisted of 50 adults (mean \pm s.d. age $=54.66 \pm 11.63$ years), including patients with a previous diagnosis of restless legs syndrome (RLS) or periodic limb movement (PLM) but without other neurological symptoms, and age-matched controls. The eligibility criterion for sleep-disturbed participants was to suffer from at least sub-threshold insomnia ( $n=26$, including 19 patients previously diagnosed with RLS/PLM and 7 controls); participants qualified as sleep-healthy subjects ( $n=24$, including 7 patients with previous RLS diagnosis and 17 controls) by scoring below the cut-off (sum score of 8 ) for subthreshold insomnia according to the insomnia severity index, which is based on the DSM-IV diagnostic criteria for insomnia.

Consistent with previous work, ${ }^{8}$ there was a significant difference in serum BDNF levels between smokers and nonsmokers $(t(47)=3.066 ; P=0.004$, ns: age, BMl and sex). Therefore, smoking was included as a covariate in subsequent analyses. Of note, participants suffering from current symptoms of insomnia

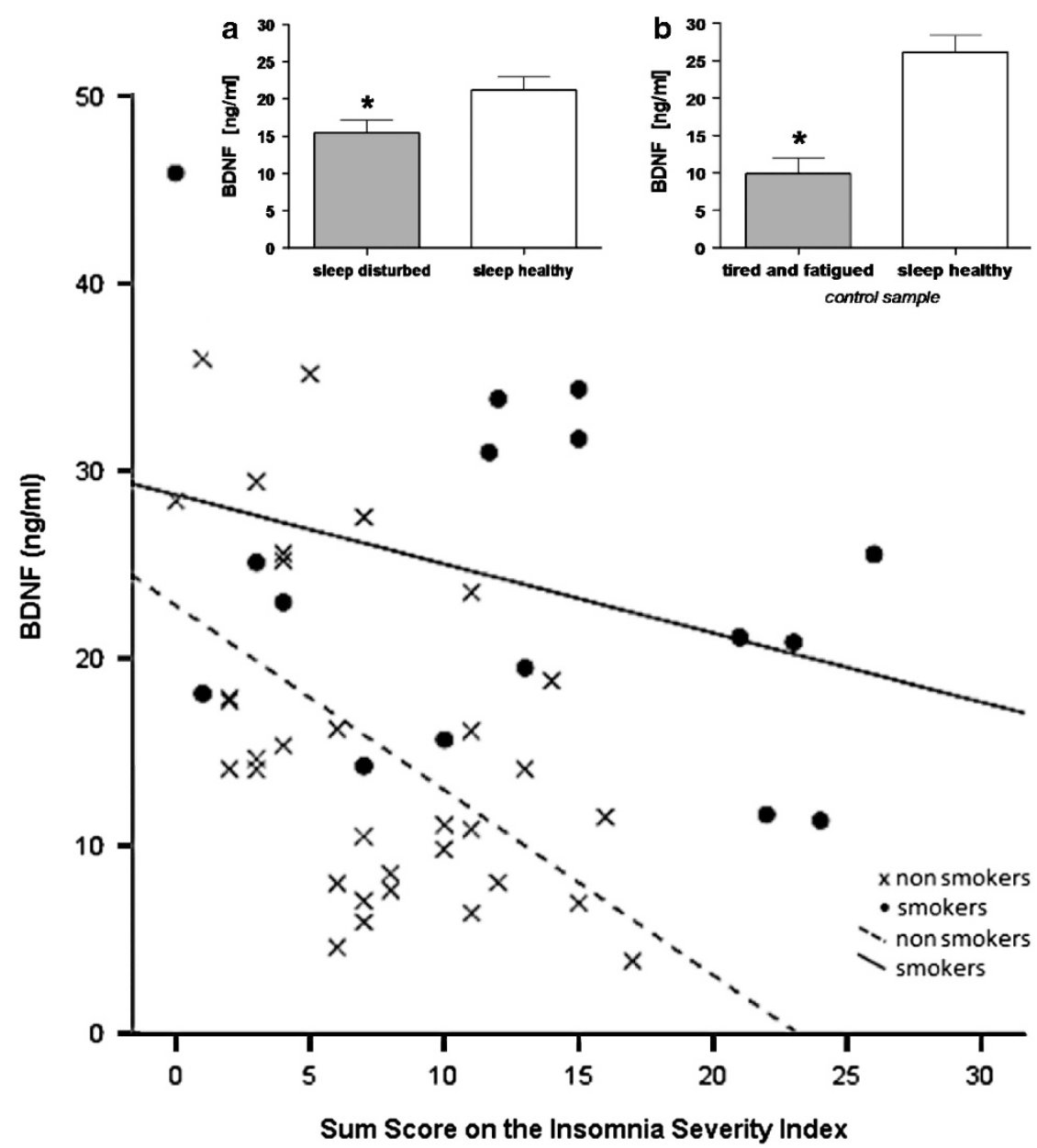

Figure 1. Correlation between serum brain-derived neurotrophic factor (BDNF) levels and insomnia severity index ratings. Analysis showed a significant correlation of BDNF levels with severity of insomnia across the sample as a whole $\left(r_{\mathrm{p}}=-0.409 ; P=0.004\right)$. Black circles represent smokers $(r=-0.331 ; P=0.21)$ and open circles represent non-smokers $(r=-0.511 ; P=0.002)$. (a) Plotted estimated means by ANOVA \pm s.e.m. of serum BDNF levels controlled for smoking of sleep-disturbed $\left(n=26 ; 15.41 \pm 1.77 \mathrm{ng} \mathrm{ml}{ }^{-1}\right)$ and sleep-helathy $(n=24$; $\left.21.15 \pm 1.80 \mathrm{ng} \mathrm{ml}^{-1}\right)$ subjects. (b) Means \pm s.e.m. of serum BDNF levels of tired and fatigued $\left(n=6 ; 9.86 \pm 2.16 \mathrm{ng} \mathrm{ml}^{-1}\right)$ and sleep-healthy $\left(n=6 ; 26.08 \pm 2.30 \mathrm{ng} \mathrm{ml}^{-1}\right)$ subjects. ${ }^{*}$ Denotes statistical significance at $P<0.05$. 
( $n=26$ ) exhibited significantly decreased serum BDNF levels compared with sleep-healthy controls $(n=24 ; \mathrm{F}(1)=5.017 ; P=0.03$; Figure 1a). In addition, serum BDNF levels were significantly correlated with severity of insomnia in all paricipants $(n=50$; $r_{\mathrm{p}}=-0.409 ; P=0.004$; Figure 1 ). There were no differences in serum BDNF levels between participants on medication (RLS medications included pramipexol and ropinirol, hypnotics, antidepressants, antipsychotics and others) and those without such drugs. Furthermore, serum BDNF levels did not differ between those with previous RLS/PLM diagnosis $(n=26$, including 19 patients with and 7 patients without insomnia symptoms) and those without such diagnoses $(n=24$, including 17 controls without and 7 with insomnia symptoms), which supports the view that serum BDNF levels are associated with sleep independently of diagnosis. We found subjective sleep impairment to be associated with lower serum BDNF levels, whereas reported good sleep was related to higher serum BDNF levels, as shown for those suffering from current insomnia compared with sleep-healthy subjects. To confirm the relevance of our preliminary findings, we investigated an additional independent control sample of adults $(n=12$, male non-smokers) that had recovered from occupational burnout and after 12 weeks of aerobic exercise training. To assess insomnia, we used the sleep-related items of the Beck Depression Inventory. Again, serum BDNF levels were significantly lower in those reporting symptoms of fatigue $(n=6)$ compared with sleephealthy subjects $(n=6 ; t=2.2625 ; P=0.025$; Figure $1 \mathrm{~b})$ and were significantly correlated $\left(n=12, r_{\mathrm{p}}=-0.639 ; P=0.025\right)$ with the symptoms of tiredness and fatigue known to reflect malfunction of sleep as the prime cause of impairments in daily life. The results from these two different samples are not strictly comparable, given the different methods of assessing disturbed sleep wake regulation as insomnia and day time fatigue, but despite or perhaps because of this, we believe that these findings support the hypothesis of an increased stress vulnerability due to sleep loss, which may lead to decreased BDNF secretion. Such a decrease might be associated with a decrease in BDNF mRNA expression in peripheral blood mononuclear cells and/or may correspond to a decline of BDNF concentration in the brain. To what extent peripheral BDNF levels correspond to brain BDNF levels remains unknown. However, the use of serum BDNF concentration as potential indicator of brain alteration is justified by extensive evidence. ${ }^{9}$ Although we report a reduction of BDNF levels linked to sleep disturbance, others have consistently shown that prolonged wakefulness as a result of sleep deprivation, which can be considered as a stressor for the brain, leads to an increase in BDNF. ${ }^{10}$ Using a bidirectional stress model as an explanatory approach, we hypothesise that chronic stress induces a deregulation of the HPA system, leading in the long term to sleep disturbance and decreased BDNF levels, whereas acute sleep deprivation, for example, one night of sleep deprivation, can be used as therapeutic intervention in some insomniac or depressed patients as a compensatory process to normalise BDNF levels.

BDNF has also been considered as a predictor of therapeutic response in major depression. However, very recent data indicate that the increase in serum levels of BDNF during antidepressant treatment appears to be confined to some but not all antidepressants and does not coincide with amelioration of clinical symptoms. ${ }^{6}$ On the basis of our results, this discrepancy might stem from changes in central BDNF concentrations, following reduced insomnia rather than depressive symptoms and antidepressants differentially influencing sleep. Thus, our preliminary findings suggest that serum BDNF levels are not associated with a specific (categorical) diagnosis, but may be associated with insomnia symptoms independent of diagnosis (dimensional). In line with this, we suggest that, when analysing serum BDNF levels in depressed patients, insomnia symptoms should be carefully controlled, as well as improvements in sleep during therapy. Further studies with a larger number of patients and a design including objective measurements of sleep, such as sleep polysomnography, should be conducted to verify the results of our exploratory investigation and to elucidate the underlying mechanisms more closely, especially the role of the stress hormone system.

\section{CONFLICT OF INTEREST}

The authors declare no conflict of interest.

M Giese ${ }^{1}$, E Unternährer ${ }^{2,3}, \mathrm{H}$ Hüttig $^{2}$, J Beck ${ }^{4}, \mathrm{~S} \mathrm{Brand}^{4}$, P Calabrese ${ }^{2}$, E Holsboer-Trachsler ${ }^{4}$ and A Eckert ${ }^{1}$

${ }^{1}$ Neurobiology Laboratory for Brain Aging and Mental Health, Psychiatric University Clinics, University of Basel, Basel, Switzerland; ${ }^{2}$ Division of Cognitive Psychology and Methodology, Department of Psychology, University of Basel, Basel, Switzerland;

${ }^{3}$ Division of Clinical Psychology and Epidemiology, Department of Psychology, University of Basel, Basel, Switzerland and

${ }^{4}$ Center for Affective, Stress and Sleep Disorders, Psychiatric Hospital of the University of Basel, Basel, Switzerland E-mail: Anne.Eckert@upkbs.ch

\section{REFERENCES}

1 Duman RS, Malberg J, Nakagawa S, D'Sa C. Biol Psychiatry 2000; 48: 732-739.

2 Faraguna U, Vyazovskiy VV, Nelson AB, Tononi G, Cirelli C. J Neurosci 2008; 28: 4088-4095.

3 Duman RS, Heninger GR, Nestler EJ. Arch Gen Psychiatry 1997; 54: 597-606.

4 Morin CM, Rodrigue S, Ivers H. Psychosom Med 2003; 65: 259-267.

5 Steiger A, Kimura M. J Psychiatr Res 2010; 44: 242-252.

6 Molendijk ML, Bus BA, Spinhoven P, Penninx BW, Kenis G, Prickaerts J et al. Mol Psychiatry 2011; 16: 1088-1095.

7 Lang UE, Hellweg R, Gallinat J. Neuropsychopharmacology 2004; 29: 795-798.

8 Bus BA, Molendijk ML, Penninx BJ, Buitelaar JK, Kenis G, Prickaerts J et al. Psychoneuroendocrinology 2011; 36: 228-239.

9 Sartorius A, Hellweg R, Litzke J, Vogt M, Dormann C, Vollmayr B et al. Pharmacopsychiatry 2009; 42: 270-276.

10 Conti B, Maier R, Barr AM, Morale MC, Lu X, Sanna PP et al. Mol Psychiatry 2007; 12: 167-189.

C) (1) $\Theta$ This work is licensed under a Creative Commons AttributionNonCommercial-NoDerivs 3.0 Unported License. To view a copy of this license, visit http://creativecommons.org/licenses/by-nc-nd/3.0/ 